\title{
Antioxidant Activity of Thymbra capitata Essential Oil in Meat-Treated Oil
}

\author{
A.D. Mohammed, M.G. Miguel and \\ M.D. Antunes \\ Faculdade de Ciências e Tecnologia \\ Universidade do Algarve \\ Campus de Gambelas \\ 8005-139 Faro \\ Portugal
}

\author{
A.C. Figueiredo, L.G. Pedro and \\ J.G. Barroso \\ Universidade de Lisboa \\ Faculdade de Ciências de Lisboa \\ DBV, IBB, Centro de Biotecnologia Vegetal \\ C2, Campo Grande \\ 1749-016 Lisboa \\ Portugal
}

Keywords: meat, essential oil, Thymbra capitata, TBARS

\begin{abstract}
Several synthetic antioxidants such as butylated hydroxyanisole (BHA), butylated hydroxytoluene (BHT), and tertiary butyl hydroquinone (TBHQ), have been used to preserve meat. Nevertheless they are considered toxic and to have carcinogenic effects. Natural products, such as those present in essential oils, have been the target of studies as alternative antioxidants, showing promising results. In addition, these natural compounds have the advantage of having consumer's acceptance. Thymbra capitata is a perennial, herbaceous shrub commonly used as a spicy herb. The essential oils isolated from this plant showed antimicrobial and antioxidant activities. The main goal of the present work was to evaluate the capacity of $T$. capitata oil to prevent lipid oxidation in bovine meat. Different concentrations of essential oil $(0,50,75$ and $100 \mathrm{mg} / 5 \mathrm{~g}$ of meat) were used in two types of bovine meat samples purchased in the local market. The protein content of both types of meat samples was $21 \%$, and lipid content ranged from $9 \%$ to $15 \%$. After covering with the oil, the two types of meat samples were stored at $-17^{\circ} \mathrm{C}$, for one month. After 1 day, 15 days and 30 days, meat samples were taken and cooked at $85^{\circ} \mathrm{C}$ for $90 \mathrm{~min}$. The samples were then submitted to the TBARS modified method, for antioxidant activity evaluation. The essential oil was able to prevent meat oxidation even after one month of storage and showed a dose-dependent activity.
\end{abstract}

\section{INTRODUCTION}

Lipid oxidation is one of the major problems occurring during processing and storage of meat. Generally, the meat oxidation is more important after cooking and refrigerated storage due to the degradation of natural antioxidants as well to the release of fatty acids and iron from haeme molecule (Estévez et al., 2007). Oxidation of fats and lipids decrease the quality and safety of foodstuffs due to the formation of peroxides, alkanes, alcohols, aldehydes and acids. One of the aldehydes that are formed after degradation of polyunsaturated fatty acids (PUFA) is malondialdehyde (MDA). Besides the rapid development of alterations of their colour, flavour, texture and nutritional value, these compounds can also contribute to the cancer, atherosclerosis, heart and allergic diseases occurrence (Bozkurt, 2007; Mielnik et al., 2008).

To overcome the fat or lipid oxidation, several synthetic antioxidants have been used such as butylated hydroxytoluene (BHT), butylated hydroxyanisole (BHA) and tertbutylhydroquinone (TBHQ). However, recent studies have revealed that these substances are not completely innocuous having been progressively banned in some European countries and Japan (Wanasundara and Shahidi, 1998; Tang et al., 2001). Moreover, they also present some instability ( $\mathrm{Du}$ and $\mathrm{Li}, 2008$ ). For these reasons the use of natural antioxidants has grown including the utilization of essential oils. Essential oils are complex mixtures of compounds belonging to diverse chemical families (terpenes, phenolics, polyacetilenic compounds, among others). 
Thymbra capitata (L.) Cav. [= Coridothymus capitatus (L.) Rchb.f, Satureja capitata L., Thymus capitatus Hoff. et Link] is a perennial, herbaceous shrub largely spread in southern Portugal commonly used as a spicy herb in some Portuguese traditional meat dishes, particularly in rabbit meat. Although thymol, carvacrol and thymol/carvacrol chemotypes are known for T. capitata oil, thymol chemotype is the most frequent in southern Apulia (Italy) (Miceli et al., 2006), and the carvacrol chemotype predominates in T. capitata from Algarve (southern Portugal) (Faleiro et al., 2005). The essential oil of T. capitata has revealed to possess antioxidant activity when evaluated by the TBARS (thiobarbituric acid reactive substances) method using egg yolk as lipid substrate (Faleiro et al., 2005). This method measures the MDA formed, after lipid hydroperoxide decomposition, which forms a pink chromophore with thiobarbituric acid (TBA). This coloured complex, which absorbs at $532 \mathrm{~nm}$, results in the condensation of 2 moles of TBA and 1 mole of malondialdehyde, in an acidic environment (Antolovich et al., 2002; Laguerre et al., 2007).

Some research has demonstrated previously the capacity of some essential oils for preventing meat oxidation (Bozkurt, 2007; Estévez et al., 2007; Du and Li, 2008; Mielnik et al., 2008). The main goal of the present work was to evaluate, by the TBARS method, the capacity of $T$. capitata oil from Portugal in preventing lipid oxidation of bovine meat.

\section{MATERIAL AND METHODS}

\section{Isolation of the Essential Oil}

The essential oil was isolated by hydrodistillation using a Clevenger-type apparatus according to the Portuguese Pharmacopoeia (2005).

\section{Isolation and Quantification of Lipids}

The extraction of lipid was carried out through the Soxhlet method using $3 \mathrm{~g}$ of bovine meat and 150-ml chloroform:methnol (3:1), for $12 \mathrm{~h}$. Lipid content was determined gravimetrically after total solvent evaporation.

\section{Quantification of Total Protein}

Two grams of bovine meat was submitted to the Kjeldahl method for the determination of overall protein concentration. Because the Kjeldahl method does not measure the protein content directly a conversion factor, $\mathrm{F}=6.25$, equivalent to $0.16 \mathrm{~g}$ nitrogen/gram of protein, were used to convert the measured nitrogen concentration to a protein concentration.

\section{Meat Sample Preparation}

Freshly bovine meat was obtained from the local market. Samples from each type of meat were homogenized with $1,1.5$ and $2 \%(\mathrm{w} / \mathrm{w})$ of essential oil of $T$. capitata. These samples were stored at $-17^{\circ} \mathrm{C}$, for one month. After 1 day, 15 days and 30 days, meat samples were taken and cooked at $85^{\circ} \mathrm{C}$ for $90 \mathrm{~min}$, then assayed for antioxidant activity using the TBARS method.

\section{TBARS Assay}

For this assay, a modified method of that reported by Ruberto and Baratta (2000) was used as described by Fasseas et al. (2007).

\section{RESULTS AND DISCUSSUION}

The essential oil, predominantly constituted by carvacrol as expected (Faleiro et al., 2005; Bounatirou et al., 2007), was used as possible antioxidant in bovine food. In the present study two types of meat was used, one being richer in lipids (15\%) after extraction by the Soxhlet method. This method was chosen because it is one of the most used for total lipid extraction from meat, although it has been demonstrated that the use of different methods results in different lipid recoveries in biological samples (Pérez- 
Palacios et al., 2008). According to these authors, such differences can be attributed to the fat and moisture content, the nature of the protein network, the presence of several additives, the technological process or the physical and chemical interactions between lipids and proteins in the meat and meat products. The lipid percentage obtained in this work was about tenfold higher than that obtained by Silva et al. (2003) for bovine meat. It is noteworthy to refer that the lipid isolation from muscle tissues used by these authors was different.

Concerning protein content, both samples presented similar percentages $(21 \%)$, within the values generally described for raw beef muscle (19-20\%) (Hurrell et al., 2006).

When evaluated by the TBARS method, the essential oil of T. capitata was able to prevent meat oxidation, even after 30 days of frozen storage (Table 1). Higher concentration of essential oil generally improved the capacity for preventing lipid oxidation. The storage time did not greatly influence the antioxidant activity of $T$. capitata oil. These results agree with those already reported by some authors that have used essential oils isolated from diverse aromatic plants on the protection of diverse type of meat or fish to lipid oxidation. Some examples include: oregano and rosemary essential oils, individually or in combination with $\alpha$-tocopheryl acetate on performance parameters and lipid oxidation of broiler meat enriched with $n$-3 PUFAs (Basmacioğlu et al., 2004); sage and rosemary essential oils on the lipid oxidative stability of refrigerated stored porcine liver pâtés (Estévez et al., 2006, 2007); rosemary essential oils on the oxidative stability of frankfurters from free range-reared Iberian pigs and intensively reared white pigs (Estévez et al., 2005); oregano and sage essential oils on porcine and bovine meat samples upon storage at $4^{\circ} \mathrm{C}$, in the raw or cooked state, over a day period (Fasseas et al., 2007); cassia essential oil on the lipid oxidation of deep-fried beef during the frying process ( $\mathrm{Du}$ and $\mathrm{Li}, 2008)$; oregano essential oil combined with light salt, modified atmosphere packaging on the shelf-life of sea-bream (Goulas and Kontominas, 2007); among many other examples.

\section{Literature Cited}

Antolovich Mm, Prenzler, P.D., Patsalides, E., McDonald, S. and Robards, K. 2002. Methods for testing antioxidant activity. The Analyst 127:183-198.

Basmacioğlu, H., Tokuşoğlu, Ö. and Ergül, M. 2004. The effect of oregano and rosemary essential oils or $\alpha$-tocopheryl acetate on performance and lipid oxidation of meat enriched with n-3 PUFA's in broiler. South African J. Animal Sci. 34:197-210.

Bounatirou, S., Smiti, S., Miguel, M.G., Faleiro, L., Rejeb, M.N., Neffati, M., Costa, M.M., Figueiredo, A.C., Barroso, J.G. and Pedro, L.G. 2007. Chemical Composition, Antioxidant and Antibacterial Activities of the Essential Oils Isolated from Tunisian Thymus capitatus Hoff. et Link. Food Chem. 105:146-155.

Bozkurt, H. 2007. Comparison of the effects of sesame and Thymbra spicata oil during the manufacturing of Turkish dry-fermented sausage. Food Control 18:149-156.

$\mathrm{Du}, \mathrm{H}$. and $\mathrm{Li}, \mathrm{H}$. 2008. Antioxidant effect of Cassia essential oil on deep-fried beef during the frying process. Meat Sci. 78:461-468.

Estévez, M., Ramírez, R., Ventanas, S. and Cava, R. 2007. Sage and rosemary essential oils versus BHT for the inhibition of lipid oxidative reactions in liver pâté. LWT 40:58-65.

Estévez, M., Ventanas, S. and Cava, R. 2006. Effect of natural and synthetic antioxidants on protein oxidation and colou rand texture changes in refrigerated stored porcine liver pâté. Meat Sci. 74:396-403.

Estévez, M., Ventanas, S., Ramírez, R. and Cava, R. 2005. Influence of the addition of rosemary essential oil on the volatiles pattern of porcine frankfurters. J. Agric. Food Chem. 53:8317-8324.

Faleiro, L., Miguel, M., Gomes, S., Costa, L., Venâncio, F., Teixeira, A., Figueiredo, A.C., Barroso, J.G. and Pedro, L.G. 2005. Antibacterial and antioxidant activities of essential oils isolated from Thymbra capitata L. (Cav.) and Origanum vulgare L. J. Agric. Food Chem. 53:8162-8168. 
Fasseas, M.K., Mountzouris, K.C., Tarantilis, P.A., Polissiou, M. and Zervas, G. 2007. Antioxidant activity in meat treated with oregano and sage essential oils. Food Chem. 106:1188-1194.

Goulas, A.E. and Kontominas, M.G. 2007. Combined effect of light salting, modifeied atmosphere packaging and oregano essential oil on the shelf-life of sea bream ((Sparus aurata): Biochemical and sensory attributes. Food Chem. 100:287-296.

Hurrell, R.F., Reddy, M.B., Juillerat, M. and Cook, J.D. 2006. Meat protein fractions enhance nonheme iron absorption in humans. J. Nutr. 136:2808-2812.

Laguerre M., Lecomte, J. and Villeneuve, P. 2007. Evaluation of the ability of antioxidants to counteract lipid oxidation: existing methods, new trends and challenges. Progr. Lipid Res. 46:244-282.

Miceli, A., Negro, C. and Tommasi, L. 2006. Essential oil variability in Thymbra capitata (L.) Cav. growing wild in Southern Apulia (Italy). Biochem. Syst. Ecol. 34:528-535.

Mielnik, M.B., Sem, S. Egelandsdal, B. and Skrede, G. 2008. By-products from herbs essential oil production as ingredient in marinade for turkey thighs. LWT 41:93-100.

Pérez-Palacios, T., Ruiz, J., Martín, D., Muriel, E. and Antequera, T. 2008. Comparison of different methods for total lipid quantification in meat and meat products. Food Chem. 110:1025-1029.

Ruberto, G. and Baratta, M.T. 2000. Antioxidant activity of selected essential oil components in two lipid model systems, Food Chem. 69:167-174.

Silva, R.G., Prado, I.N., Matsushita, M., Visentainer, J.V., Moreira, F.B. and Souza, N.E. 2003. Diets and genetic groups effects on the muscle composition and fatty acid profiles of heifers fattened in feedlot. Acta Scientiarum. Technol. 25:71-76.

Tang, S., Kerry, J.P., Sheehan, D., Buckley, D.J. and Morrissey, P.A. 2001. Antioxidative effect of added tea catechins on susceptibility of cooked red meat, poultry and fish patties to lipid oxidation. Food Res. Int. 34:651-657.

Wanasundara, U.N. and Shahidi, F. 1998. Antioxidant and pro-oxidant activity of green tea extracts in marine oils. Food Chem. 63:335-342.

\section{$\underline{\text { Tables }}$}

Table 1. Antioxidant activity of T. capitata essential oil on bovine meat, expressed as percentage of inhibition, evaluated through the TBARS method.

\begin{tabular}{lcccccc}
\hline Oil $\begin{array}{l}\text { Concentration } \\
\%(w / w)\end{array}$ & 1 day & 15 days & 30 days & 1 day & 15 days & 30 days \\
\cline { 2 - 7 } & 48.9 & - & 28.9 & 46.7 & 43.2 & 14.6 \\
1.5 & - & 25.7 & 44.5 & 60.3 & 60.1 & 74.4 \\
2 & 79.7 & 55.0 & 74.6 & 69.2 & 70.6 & 74.6 \\
\hline
\end{tabular}

